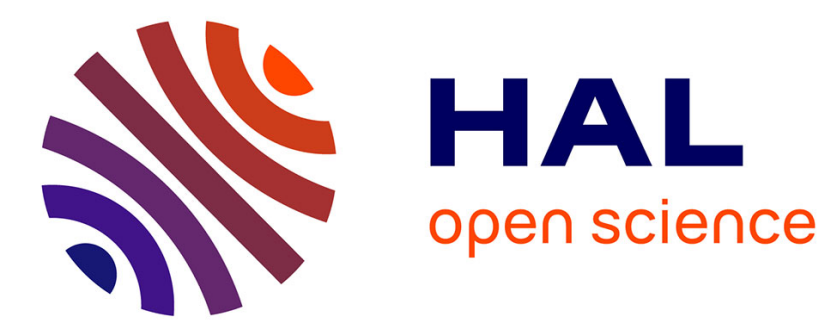

\title{
Antoine Léon (1921-1998) : un précurseur de l'orientation éducative
}

Pierre Roche

\section{To cite this version:}

Pierre Roche. Antoine Léon (1921-1998) : un précurseur de l'orientation éducative. L'Orientation scolaire et professionnelle, 2019, 48 (2), pp.231-241. 10.4000/osp.10747 . hal-03449942

\section{HAL Id: hal-03449942 https://hal-cnam.archives-ouvertes.fr/hal-03449942}

Submitted on 25 Nov 2021

HAL is a multi-disciplinary open access archive for the deposit and dissemination of scientific research documents, whether they are published or not. The documents may come from teaching and research institutions in France or abroad, or from public or private research centers.
L'archive ouverte pluridisciplinaire HAL, est destinée au dépôt et à la diffusion de documents scientifiques de niveau recherche, publiés ou non, émanant des établissements d'enseignement et de recherche français ou étrangers, des laboratoires publics ou privés.

$$
\text { Copyright }
$$




\section{O.S.P.}

\section{L'orientation scolaire et professionnelle}

$48 / 2$ | 2019

Regards sur l'histoire de l'orientation en France

\section{Antoine Léon (1921-1998) : un précurseur de l'orientation éducative}

Antoine Léon (1921-1998): a precursor of educative guidance

\section{Pierre Roche}

\section{(2) OpenEdition}

\section{Journals}

Édition électronique

URL : https://journals.openedition.org/osp/10747

DOI : 10.4000/osp. 10747

ISSN : 2104-3795

Éditeur

Institut national d'étude du travail et d'orientation professionnelle (INETOP)

\section{Édition imprimée}

Date de publication : 1 juin 2019

Pagination : 231-241

ISSN : 0249-6739

Ce document vous est offert par Conservatoire national des arts et métiers (Cnam)

\section{le cnam}

Référence électronique

Pierre Roche, «Antoine Léon (1921-1998) : un précurseur de l'orientation éducative », L'orientation scolaire et professionnelle [En ligne], 48/2 | 2019, mis en ligne le 01 juin 2021, consulté le 25 novembre 2021. URL : http://journals.openedition.org/osp/10747 ; DOI : https://doi.org/10.4000/osp.10747

Ce document a été généré automatiquement le 25 novembre 2021.

(c) Tous droits réservés 


\section{Antoine Léon (1921-1998) : un précurseur de l'orientation éducative}

Antoine Léon (1921-1998): a precursor of educative guidance

Pierre Roche

\section{Introduction}

1 Cette page d'histoire ${ }^{1}$ sera consacrée à éclairer la place du conseiller d'orientation professionnelle Antoine Léon dans l'évolution de l'orientation. Cela nous conduira à nous intéresser à l'itinéraire d'Antoine Léon, aux continuités et ruptures dans la vie de ce conseiller d'orientation professionnelle, confronté à deux maîtres renommés, $\mathrm{H}$. Wallon et $\mathrm{H}$. Piéron, tous deux professeurs au Collège de France, dans une époque très politique. L'appréhender nous amènera à évoquer les grandes étapes des cinquante ans d'une vie de recherches et de combats, dont la richesse et la fécondité apparaissent aujourd'hui.

2 Le contexte historique joue un rôle important dans ce parcours. L'essentiel de l'œuvre d'Antoine Léon, en psychologie et dans le domaine des sciences de l'éducation, se situe, à part presque égale, autour de mai 1968, entre 1950 et 1985 . Il a vécu le Front populaire, l'Occupation, la Libération et, du milieu du siècle aux premières années du septennat de François Mitterrand, les années de la guerre froide, les désillusions des communistes après la publication du rapport Khroutchev de 1956, les guerres coloniales et l'arrivée au pouvoir de de Gaulle, les Trente Glorieuses, l'union de la gauche, le Programme commun et l'arrivée de la gauche au pouvoir en 1981.

3 L'influence du contexte de la guerre froide sur la perception de l'œuvre de Léon est bien décrite par le témoignage de notre collègue Maurice Athané2, qui m'écrivait en 1999: "C'était une époque où l'on pouvait supposer que le parti (communiste) prendrait le pouvoir, tant était grand le prestige de l'URSS, principal vainqueur de 
l'Allemagne, et aussi vu l'action efficace et prépondérante des communistes dans la Résistance ». Il évoquait aussi «l'anti-marxisme diffus qui imprégnait les esprits dans les années 1950 et qui explique en partie la méfiance, voire l'hostilité dont fut alors victime l'ouvrage d'A. Léon sur la psycho-pédagogie de l'orientation. Les idées qu'il présentait nous paraissent aujourd'hui novatrices et fécondes. Mais l'engagement militant de l'auteur au Parti communiste français les rendait alors suspectes. [...] toute opinion exprimée par un communiste était considérée comme étroitement liée à des directives émanant de l'Union soviétique. Comment ne pas douter de leur objectivité ?».

\section{Le parcours d'Antoine Léon}

4 Né le $1^{\text {er }}$ octobre 1921 dans la Casbah d'Alger, Antoine Léon entre à l'École normale à l'issue d'une brillante scolarité. Il participe de 1943 à 1945 aux différentes campagnes militaires de la Seconde Guerre mondiale (Afrique du Nord, Corse, France, Allemagne) et adhère en août 1944 au Parti communiste français (PCF). Démobilisé en septembre 1945, il entre en 1946 à l'Institut national d'études du travail et d'orientation professionnelle (Inetop) à Paris et y prépare le diplôme de conseiller d'orientation professionnelle. Il intègre ensuite le service de recherches de l'Inetop que dirige alors Mme Piéron, puis à partir de 1950 Maurice Reuchlin, pour y travailler de 1947 à 1957.

Il relie son engagement communiste militant à son activité professionnelle et devient rapidement la principale figure des communistes de l'orientation professionnelle. Ainsi participe-t-il très activement à l'élaboration de positions marxistes très critiques envers la psychotechnique et les tests et à la publication d'articles exposant ces positions dans les revues marxistes La Raison et La Pensée. Il définit en 1955 le cadre conceptuel d'une nouvelle approche de l'orientation scolaire et professionnelle qu'il expose tout d'abord dans le Binop, en faisant le compte-rendu de ses recherches, puis en 1957 dans un ouvrage intitulé Psychopédagogie de l'orientation professionnelle préfacé par H. Wallon (Léon, 1957). Dans cette approche, il propose de substituer une conception éducative, formatrice, à la conception révélatrice, diagnostique de l'orientation professionnelle fondée sur une théorie des aptitudes de Piéron.

6 Selon lui, il faut définir les objectifs éducatifs et les formes pédagogiques de l'orientation professionnelle. Aller vers l'autodétermination du jeune, c'est l'informer et combattre ses préjugés, accroître la plasticité de ses goûts professionnels et sa liberté, comme son application dans la scolarité, lui donner des perspectives. Cela passe par l'intégration des moyens d'information dans un programme annuel, par l'introduction d'une initiation technique et sociale dans les programmes, par l'aménagement des travaux manuels, par des films et des visites d'établissements scolaires et d'entreprises, par des leçons sur la formation et la vie professionnelles.

7 En 1957, il quitte l'Inetop et enseigne ensuite pendant trois ans la psychologie à l'université de Toulouse avec Philippe Malrieu ${ }^{3}$, puis, recruté au CNRS, il prépare en quelques années deux thèses, toutes deux soutenues et publiées : Formation générale et apprentissage du métier (1965) et La Révolution française et l'éducation technique (1968). Dès ce moment, et ce sera le cas durant toute sa carrière universitaire, il s'intéressera à deux domaines de recherche : la psychopédagogie et l'histoire de l'enseignement et de l'éducation. 
De 1964 à 1968, il est maître-assistant de pédagogie à la faculté des lettres et sciences humaines de Paris. Il publie successivement en 1961, aux Presses universitaires de France dans la collection "Que-sais-je? » Histoire de l'éducation technique (1961) et en 1967, Histoire de l'enseignement en France.

9 Maître de conférences de 1968 à 1970 et professeur à l'université Paris V de 1970 à 1985, il publie plusieurs livres pionniers à visée pédagogique sur des thématiques trop peu développées: Psychopédagogie des adultes (1971), Introduction à l'histoire des faits éducatifs (1980), Histoire de l'éducation populaire (1983), Histoire de l'éducation aujourd'hui, et en collaboration: La formation des maîtres (1974), Enseignement technique et formation permanente (1976), Manuel de psychopédagogie expérimentale (1977), Questions réponses sur l'enseignement technique court (1978).

10 Ce n'est qu'en 1979, à la fin de sa carrière universitaire, et en 1991, alors retraité, qu'il commentera ses engagements et son œuvre dans deux articles portant sur les travaux d'Henri Wallon et sur les activités des communistes de l'OP dans ces années 1950. Ces articles sont essentiels pour comprendre sa trajectoire intellectuelle et ses liens à Wallon, à Piéron et au communisme.

\section{Les relations de Léon avec Wallon et Piéron}

11 La filiation scientifique et l'attachement de Léon à Wallon sont clairs et explicites. Dans l'ouvrage Psychopédagogie de l'orientation professionnelle, qu'il publie en 1957, il écrit ainsi : "Notre reconnaissance va enfin à M. le Professeur Henri Wallon dont l'œuvre psychologique et pédagogique a été un guide constant pour l'élaboration de nos idées ». On remarquera que dans les pages de ce livre Henri Wallon est nommément cité à quinze reprises (en comparaison, Henri Piéron ne l'est que deux fois).

larticle qu'il publie en 1979 dans Paedagogica historica, revue internationale d'histoire de la pédagogie, et qui est intitulé Psychopédagogie scientifique et humanisme dans l'œuvre d'Henri Wallon (1879-1962), il reconnait de nouveau l'ampleur de sa dette envers Wallon : «Il ne saurait, bien entendu, être question de présenter ici tout ce que nous devons à l'œuvre d'Henri Wallon ».

13 La nature de cet apport décisif de Wallon dans l'œuvre de Léon n'est pas explicitée par lui-même. En analysant ses écrits, nous parlerons d'un marxisme élaboré durant une vie de recherches incluant une certaine conception du fait scientifique et de la méthode pour le saisir où s'opposent les attributs et les relations, la substance et l'accident, la constatation et l'éducation ${ }^{4}$, avec pour modèle le psychologue scolaire (Roche, 2019).

Les relations à Piéron sont plus complexes. Si l'on suit le professeur Mira y Lopez dans sa préface à l'ouvrage de Léon (1957) dans sa traduction brésilienne, les relations de Léon avec Piéron étaient excellentes: «Le professeur Antoine Léon a été le disciple préféré des maîtres H. Piéron et Henri Wallon » écrit-il (Mira y Lopez, 1958). Léon luimême donne une vision positive de sa relation avec Piéron en novembre 1965, dans son exposé de présentation de sa thèse, où il reconnaît l'apport conjoint de Wallon et de Piéron à sa formation: "Si je devais situer ces travaux par rapport aux grandes divisions de la Psychologie, je dirais qu'ils s'inscrivent à la fois dans la perspective de la Psychologie génétique et dans celle de la Psychologie du comportement. Il est vrai que j'ai fait mon apprentissage de chercheur dans une maison, l'Institut national 
d'orientation professionnelle, où ces deux courants étaient représentés respectivement par Henri Wallon et Henri Piéron » (Léon, 1965).

Cependant, les relations avec Piéron furent essentiellement conflictuelles, du fait de leur divergence de vue sur l'orientation.

\section{Trois épisodes marquants de l'opposition Piéron-Léon}

16 Trois situations attestent d'une rébellion croissante des communistes et d'Antoine Léon au modèle développé par Piéron.

17 Comme en témoignait mon ami et collègue Jean Cnudde, né en 1930, militant du Snes, qui m'écrivait, avec une distance amusée qui n'enlève rien à la véracité de son témoignage, "à l'Inetop, les étudiants de la cellule communiste se réunissaient autour de Léon qui avait la réputation d'un dangereux subversif auprès de la direction de l'Institut ${ }^{5}$ ».

18 Une première confrontation a lieu entre les étudiants communistes en formation à l'Inetop et $\mathrm{H}$. Piéron, directeur de l'Institut, et à l'initiative de ce dernier, entre novembre 1951 et janvier 1952.

19 Les étudiants communistes en formation à l'Inetop, regroupés en cellule, éditent un journal intitulé $\mathrm{La}$ Boussole dans lequel ils développent leurs réflexions sur l'orientation et sur leur formation. Henri Piéron va réunir le 8 janvier 1952 dans le grand amphithéatre de l'Institut les deux promotions des étudiants et va leur lire ce passage de La Boussole, qu'il ne peut bien évidemment admettre : "Nous avons voulu seulement mettre en garde les Inopiens soucieux de leur avenir contre une étroite spécialisation technique limitée à l'utilisation des tests. Une telle spécialisation les mènerait inéluctablement à une impasse, car il est certain que les "tests d'aptitudes" ne peuvent se concevoir et exister que comme un mauvais palliatif à certaines des contradictions internes du capitalisme en décomposition. Leur disparition suivra inévitablement à brève échéance celle du régime qui leur a donné naissance. Nous engageons, en conclusion, chaque Inopien, qui dispose de deux ans de vie parisienne, avant de commencer à orienter du mieux qu'il pourra les enfants d'une sous-préfecture, à utiliser son temps au maximum pour acquérir des connaissances dans tel ou tel domaine de son goût en tenant compte de la réorientation qu'il devra nécessairement s'imposer dans un avenir que nous, communistes, et même conseillers d'OP communistes, nous espérons très proche ${ }^{6} »$. Piéron va ensuite intimer l'ordre aux étudiants concernés de se démettre ou de se renier. Mais il n'y aura pas de défection, ni de démission, et chacun restera sur ses positions.

20 Un second fait significatif intervient lors des Journées nationales d'études des intellectuels communistes les 29 et 30 mars $1953^{6}$ avec la prise de parole d'Antoine Léon en qualité de porte-parole des conseillers d'OP communistes, devant 600 intellectuels communistes.

21 Son exposé met en avant trois lignes politiques en partie contradictoires. La première porte à la fois une condamnation du modèle sur lequel s'appuie la formation dispensée à l'Inetop et introduit la conception éducative de l'orientation qui lui est propre: « Poser nos problèmes en termes de formation et de rattrapage et non en termes de détection d'aptitudes immuables, doit nous permettre d'envisager, sinon d'obtenir, des solutions plus saines que celles qui résulteraient d'une attitude techniciste». Mais 
l'essentiel de son intervention, une deuxième ligne, est une dénonciation de l'orientation professionnelle telle qu'elle se pratique alors dans le cadre d'une société capitaliste : « Si, dans notre métier, nous apportons une certaine aide aux adolescents en les informant sur les possibilités d'apprentissage et de placement et en leur évitant des erreurs d'aiguillage, nous ne devons pas perdre de vue que la réussite professionnelle de la grande majorité de la jeunesse se trouve limitée par la loi du profit maximum. Les quelques exemples de sauvetage individuels que chacun de nous peut rassembler ne doivent pas masquer les effets de cette loi ». Enfin, une troisième ligne, alors dominante, se fait jour quand il fait état des activités d'une conseillère communiste de province qui, dans un premier temps, déconseillait aux élèves de niveau faible aux tests de se présenter au concours d'entrée du centre d'apprentissage, ce qui avait suscité une menace de fermeture de l'établissement et qui ensuite, dans un deuxième temps, avait dirigé vers l'établissement tous les jeunes sans tenir compte de leurs résultats aux tests et a organisé une action de "masse " contre la menace de fermeture. Ce qui avait conduit la municipalité à envisager la création de deux centres d'apprentissage.

Un troisième temps de ce conflit, le plus violent, se produit ensuite, en 1954, quand $\mathrm{H}$. Piéron dénonce publiquement et nommément $\mathrm{A}$. Léon dans un article qu'il publie dans le Binop intitulé : «Le rôle d'un conseiller d'OP ne doit pas se confondre avec celui d'un éducateur » (Piéron, 1954).

Ce texte de moins de trois pages est capital. Il résume et éclaire toute l'histoire de l'orientation scolaire et professionnelle du $\mathrm{XX}^{\mathrm{e}}$ siècle sous la forme d'un rappel de l'évolution de la profession et un tableau des courants d'idées qui s'affrontent à son sujet.

Piéron attaque l'approche de Léon en ces termes : «Mais voici que se développe, parmi certains de ces anciens instituteurs, sous certaines influences, un mouvement qui risque de saper les bases mêmes de la profession, et de fournir une aide à ceux qui, pour des raisons diverses et parfois opposées n'ont pas renoncé à obtenir sa suppression ». Pour H. Piéron, fondateur d'une approche scientifique de l'orientation professionnelle, le mouvement initié par Léon, au sein même du service de recherches de l'Inetop, représente un retour en arrière et en rien un progrès. Ainsi, pour lui, le communiste devient un réactionnaire et un disciple des premiers temps de l'OP, et par exemple, de Mauvezin et de sa Rose des métiers. Il écrit : « Il s'agirait de réduire le rôle du conseiller à une tâche d'information et d'éducation, en un retour à ce stade initial où toute technicité paraissait inutile ». Pour lui, cette régression est imputable au marxisme, qu'il décrit comme "une doctrine attribuant aux influences sociales une portée qui rendrait négligeables les différences constitutives ».

Le texte d'H. Piéron est suivi d'une note d'A. Léon dans laquelle ce dernier marque son émotion d'être accusé de "saper les bases mêmes de la profession " et invite à un débat argumenté à partir des résultats de ses recherches et de celles de plusieurs de ses collègues.

\section{Léon et la position du Parti communiste français sur l'orientation}

Accusé de porter la position du PCF, A. Léon va pourtant se distancier de celle-ci. 
lit et ce sera sur une ligne qui correspond à celle défendue par A. Léon : «Il s'agit, en effet, avant tout, de savoir dans quelles conditions s'effectuera la transformation de l'OP en tenant compte des contradictions quotidiennes auxquelles se heurtent les praticiens dans l'exercice de leur profession. [...] la véritable défense de l'OP ne s'identifie pas avec la préservation jalouse de sa structure et de ses méthodes actuelles ». Ce qui démontre que la ligne dominante de contestation absolue ne peut se justifier que sous la forme d'une voie éducative.

\section{La reconnaissance du rôle précurseur d'Antoine Léon}

En 1997, Jean Guichard, professeur des universités et alors directeur de l'Inetop, écrivait à propos de l'ouvrage Psychopédagogie de l'orientation professionnelle : "C'est en 1957 que fut systématiquement développé - certainement pour la première fois en France - une conception radicalement nouvelle de l'orientation. [...] Cet ouvrage est à l'orientation professionnelle ce que fut la révolution copernicienne à l'astronomie : [...] Les idées fondamentales de l'orientation éducative d'aujourd'hui se trouvent toutes dans la psychopédagogie de Léon » (Guichard, 1997, p. 41).

L'orientation scolaire et professionnelle, 48/2 | 2019 
Lors de la parution de cet article, j'en avais adressé une copie à A. Léon. Il m'avait immédiatement répondu le 16 janvier 1998, peu avant sa mort : «Antoine Léon vous remercie vivement d'avoir pensé à lui adresser une copie de l'article de Jean Guichard, dont il ignorait qu'il fût l'actuel directeur de l'Inetop. Il ne vous étonnera pas en vous disant qu'il a été agréablement surpris par les éloges, sans doute excessifs, visant un ouvrage publié il y a 40 ans et qui représente le terme de la seconde étape de son parcours professionnel. Il vous assure de son fidèle et cordial souvenir ».

Lors de ses obsèques, au crématorium de Montfermeil, le 13 mars, son frère Louis, récemment décédé, m'a dit la joie qui avait été la sienne de voir reconnus, quarante ans après, ses travaux qui passaient en 1954 pour Henri Piéron comme destructeurs de la profession.

Nous pouvons ainsi aujourd'hui reconnaître combien Antoine Léon, doublement rebelle vis-à-vis de la ligne scientifique de Piéron à l'Inetop et de la ligne politique et idéologique dominante du PCF, a contribué à l'histoire moderne de l'orientation.

\section{BIBLIOGRAPHIE}

Collectif (1953). Documents des Journées nationales d'études des intellectuels communistes (Ivry, 29-30 mars 1953). La Nouvelle Critique, 45, 125-368.

Fougeyrollas, P. (1950). La position de Parti dans les sciences humaines. La Nouvelle Critique, 15, 60-69.

Fougeyrollas, P. (1951). De la psychotechnique à la sociologie policière. La Nouvelle Critique, 28, 25-46.

Georges, G., Hannoun, H., Léon, A., \& Toraille, R. (1974). La formation des maîtres, Paris : Éditions ESF.

Guichard, J. (1997), L'Éducation à l'orientation, Éducations, 11, 41-43.

Léon, A. (1955). La place de l'information dans l'orientation professionnelle en France. Bulletin de Psychologie, 4, 220-231.

Léon, A. (1957). Psychopédagogie de l'orientation professionnelle. Paris : Presses universitaires de France.

Léon, A. (1961). Histoire de l'éducation technique. Paris : Presses universitaires de France. Collection «Que-sais-je?».

Léon, A. (1965). Formation générale et apprentissage du métier, Bulletin de psychologie, 245- XIx, 2-5, 182-186.

Léon, A. (1967). Histoire de l'enseignement en France. Paris : Presses universitaires de France. Collection « Que-sais-je?».

Léon, A. (1971). Psychopédagogie des adultes. Paris : Presses universitaires de France.

Léon, A. \& Chassignat, A. (1976). Enseignement technique et formation permanente. Revue française de pédagogie, 37, 45-48. 
Léon, A. (Dir.). (1977). Manuel de psychopédagogie expérimentale. Paris : Presses universitaires de France.

Léon, A. (1979). Psychopédagogie scientifique et humanisme dans l'œuvre d'Henri Wallon (1879-1962), Paedagogica historica, 19(1), 132145.

Léon, A. (1980). Introduction à l'histoire des faits éducatifs. Paris : Presses universitaires de France.

Léon, A. (1983). Histoire de l'éducation populaire. Paris : Fernand Nathan.

Léon, A. (1986). Histoire de l'éducation aujourd'hui. Paris : Unesco ; Lausanne : Delachaux et Niestlé.

Léon, A. (1991). Note sur l'histoire de l'orientation professionnelle en France. Histoire de

l'éducation, 49, 89-98.

Léon, A. (Ed.). (1978). Questions réponses sur l'enseignement technique court. Paris : ESF éditeur.

Maniez, J.-P., \& Pernin, C. (1988). Un métier moderne. Le conseiller d'orientation. Paris : L'Harmattan.

Piéron, H. (1954). Le rôle d'un conseiller d'OP ne doit pas se confondre avec celui d'un éducateur. Note de A. Léon. Binop, 3, 133-136.

\section{NOTES}

1. Une première version de ce texte, intitulée « Un disciple et rebelle à l'Inetop durant la guerre froide ", a été présentée le 14 septembre 2018 lors du séminaire organisé par l'Inetop pour son $90^{\mathrm{e}}$ anniversaire.

2. Maurice Athané, né en 1920, promotion 1946-1948 de l'Inetop.

3. Philippe Malrieu (1912-2005).

4. J'ai récemment repris cette question dans ma contribution intitulée « Antoine Léon et la psychopédagogie des adultes » dans l'ouvrage Psychologies pour la formation, dirigé par Philippe Carré et Patrick Mayen paru chez Dunod en juin 2019.

5. Lettre de J. Cnudde du 19 janvier $1999{ }^{6}$ La Boussole, n 60, 27 novembre 1951.

6. La Nouvelle Critique, $n^{\circ} 45$, avril-mai 1953.

\section{RÉSUMÉS}

Antoine Léon (1921-1998), conseiller d'orientation professionnelle au service de recherches de l'Inetop entre 1947 et 1957, disciple d'Henri Wallon et militant communiste, élabore, avec plusieurs de ses collègues une orientation professionnelle éducative en opposition à la conception de l'orientation professionnelle alors dominante en France, portée par Henri Piéron, basée sur l'évaluation des aptitudes par les tests psychotechniques. Il prend également ses distances avec la ligne politique de son parti. Il devint professeur à la Sorbonne en sciences de l'éducation et spécialiste en histoire et psychologie, au terme d'une carrière prolifique de près de cinquante ans marquée par la publication d'une quinzaine d'ouvrages et 160 articles. Son œuvre rebelle, jugée à l'époque destructrice par Piéron, posera pourtant les bases d'une approche éducative en orientation qui s'imposera plusieurs décennies plus tard. 
Antoine Léon (1921-1998), was a vocational guidance counselor at the research department of Inetop between 1947 and 1957. Mentored by Henri Wallon and active communist, he elaborates, with several of his colleagues, a pedagogical guidance opposed to the approach, then dominating in France, driven by Henri Piéron, based on the evaluation of abilities by psychotechnical tests. He distanced himself from the political line of his party. He became a professor of education at Sorbonne university and a specialist in history and psychology, after a prolific career of almost fifty years marked by the publication of fifteen books and one hundred and sixty articles. His work, strongly criticized by Pieron, lay the foundation for a pedagogical approach that will be imposed several decades later.

INDEX

Keywords : vocational guidance, Inetop, education, Leon, Piéron

Mots-clés : orientation professionnelle, Inetop, éducation, Léon, Piéron

\section{AUTEUR}

\section{PIERRE ROCHE}

Président du Groupe de recherches sur l'évolution de l'orientation scolaire et professionnelle (GREO) - Inetop, Cnam 